\title{
Note on spectral semistar operations, II
}

\author{
Ryûki MATsUDA*
}

\begin{abstract}
We study an infinite dimensional and non-local Prüfer domain $D$, and show that there are many non-spectral semistar operations on $D$.
\end{abstract}

\section{Introduction}

This is a continuation of [M3]. Let $D$ be an integral domain with quotient field $K$. Let $\overline{\mathrm{F}}(D)$ be the set of non-zero $D$-submodules of $K$, let $\mathrm{F}(D)$ be the set of non-zero fractional ideals of $D$, i.e., $E \in \mathrm{F}(D)$ if $E \in \overline{\mathrm{F}}(D)$ and there is an element $d \in D \backslash\{0\}$ such that $d E \subseteq D$. A semistar operation on $D$ is a mapping $\star: \overline{\mathrm{F}}(D) \longrightarrow \overline{\mathrm{F}}(D)$, $E \longmapsto E^{\star}$, such that for every $x \in K \backslash\{0\}$ and for every $E, F \in \overline{\mathrm{F}}(D)$, the following conditions hold: $(x E)^{\star}=x E^{\star} ; E \subseteq F$ implies $E^{\star} \subseteq F^{\star} ; E \subseteq E^{\star}$, and $\left(E^{\star}\right)^{\star}=E^{\star}$. The identity mapping $E \longmapsto E^{\mathrm{d}}=E$ on $\overline{\mathrm{F}}(D)$ is a semistar operation called the dsemistar operation on $D$. For every $\Delta \subseteq \operatorname{Spec}(D)$, the mapping $E \longmapsto \cap_{P \in \Delta} E D_{P}$ is a semistar operation on $D$, and is denoted by $\star_{\Delta}$. A semistar operation $\star$ on $D$ is called spectral if there is a subset $\Delta \subseteq \operatorname{Spec}(D)$ such that $\star=\star_{\Delta}$. G. Picozza [P, Corollary 4.13] proved the following:

Let $D$ be a local domain, i.e., $D$ has only one maximal ideal. Then every semistar operation on $D$ is spectral if and only if $D$ is a discrete valuation domain. In [M3, Theorem 1.1], we proved the following:

Let $D$ be a finite dimensional domain. Then every semistar operation on $D$ is spectral if and only if $D$ is a discrete valuation domain.

A valuation domain $V$ with value group $\Gamma$ is called discrete if, for every pair $H_{1}, H_{2}$ of adjacent convex subgroups of $\Gamma$ (say, $H_{1} \varsubsetneqq H_{2}$ ), the ordered factor group $\frac{H_{2}}{H_{1}}$ is order isomorphic with $\boldsymbol{Z}$. Next, for totally ordered abelian additive groups $A$ and $B$, we introduce the lexicographic order on $A \oplus B$; If $x=a+b \in A \oplus B$ with $b>0$, then $x>0$.

We posed a question in [M3]. Thus, let $A_{1}, A_{2}, B$ be non-zero totally ordered abelian additive groups. Let $v_{0}: A_{1} \oplus A_{2} \oplus B \longrightarrow A_{1} \oplus B$ be the projection, let $w_{0}: A_{1} \oplus A_{2} \oplus B \longrightarrow A_{2} \oplus B$ be the projection, and let $u_{0}: A_{1} \oplus A_{2} \oplus B \longrightarrow B$ be the projection. Let $k$ be a field, let $k\left[X ; A_{1} \oplus A_{2} \oplus B\right]$ be the group ring of $A_{1} \oplus A_{2} \oplus B$ over $k$, and let $K$ be the quotient field of $k\left[X ; A_{1} \oplus A_{2} \oplus B\right]$. Let $f=\sum a_{i} X^{\alpha_{i}} \in$

Received 8 June 2012; revised 13 October 2012

2000 Mathematics Subject Classification. 13A15

Key Words and Phrases. star operation, semistar operation.

*Professor Emeritus, Ibaraki University, Mito, Ibaraki 310-8512, Japan (rmazda@adagio.ocn.ne.jp) 
$k\left[X ; A_{1} \oplus A_{2} \oplus B\right]$ with $a_{i} \neq 0$ for every $i$, and $\alpha_{i} \neq \alpha_{j}$ for every $i \neq j$. We set $v(f)=\min _{i} v_{0}\left(\alpha_{i}\right)$, then $v$ defines a valuation $v$ on $K$ with value group $A_{1} \oplus B$. Similarly, we have a valuation $w$ with value group $A_{2} \oplus B$. Let $V$ (resp., $W$ ) be the valuation domain of $v$ (resp., $w$ ), and set $D:=V \cap W$. Our question is the following:

[M3, Question 3.4]: Let $A$ be the set of positive real numbers. For every $r \in A$ and for every $k \in \boldsymbol{Z}$, let $e_{r}$ (resp., $\epsilon_{r}$ ) be a symbol, let $k e_{r}$ (resp., $k \epsilon_{r}$ ) be a copy of $k$, and let $\boldsymbol{Z} e_{r}$ (resp., $\boldsymbol{Z} \epsilon_{r}$ ) be a copy of $\boldsymbol{Z}$. Now set $A_{1}:=\oplus_{r \in A} \boldsymbol{Z} e_{r}$, set $A_{2}:=\oplus_{r \in A}$ $\boldsymbol{Z} \epsilon_{r}$, and set $B:=\boldsymbol{Z} e$, where $e$ is another symbol. Then, is every semistar operation on $D$ spectral? In this paper, we study the question and give a negative answer.

\section{A Theorem of E. Houston}

Let $D$ be a domain with quotient field $K$. A star operation on $D$ is a mapping $\star: \mathrm{F}(D) \longrightarrow \mathrm{F}(D), E \longmapsto E^{\star}$, such that for every $x \in K \backslash\{0\}$ and for every $E, F \in \mathrm{F}(D)$, the following conditions hold: $D^{\star}=D ;(x E)^{\star}=x E^{\star} ; E \subseteq F$ implies $E^{\star} \subseteq F^{\star} ; E \subseteq E^{\star}$, and $\left(E^{\star}\right)^{\star}=E^{\star}$.

For a Prüfer domain $D$, call two maximal ideals $M, N$ of $D$ dependent if $M \cap N$ contains a non-zero prime ideal. This defines an equivalence relation on the set of maximal ideals of $D$, and we denote by $\left\{A_{\lambda} \mid \lambda \in \Lambda\right\}$ the corresponding partition. For each $\lambda \in \Lambda$, denote by $P_{\lambda}$ the largest prime ideal of $D$ contained in $\cap_{M \in A_{\lambda}} M$, and let $S_{\lambda}=\cap_{M \in A_{\lambda}} D_{M}$. Recently, E. Houston proved the following,

Theorem $1([\mathrm{H}])$. An integrally closed domain $D$ admits only finitely many star operations if and only if $D$ is a Prüfer domain which satisfies the following conditions: (1) Each non-zero element of $D$ is contained in only finitely many maximal ideals, (2) $\left|A_{\lambda}\right|=1$ for almost all $\lambda \in \Lambda$, (3) $\left|\operatorname{Spec}\left(\frac{D}{P_{\lambda}}\right)\right|<\infty$ for each $\lambda \in \Lambda$, and (4) $D$ has only finitely many non-invertible maximal ideals.

Moreover, under these conditions, if we denote by $\operatorname{Star}(D)$ the set of star operations on $D$, then $|\operatorname{Star}(D)|=\prod_{\lambda \in \Lambda}\left|\operatorname{Star}\left(S_{\lambda}\right)\right|$.

For semistar operations, we have the following,

Theorem 2 ([M2, Section 5, (5.2), Theorem]). An integrally closed domain $D$ admits only finitely many semistar operations if and only if $D$ is a finite dimensional Prüfer domain which has only finitely many maximal ideals.

\section{An Answer to Question}

Throughout the section, as in $\S 0$, let $A$ be the set of positive real numbers, for every $r \in A$ and for every $k \in \boldsymbol{Z}$, let $e_{r}$ (resp., $\epsilon_{r}$ ) be a symbol, let $k e_{r}$ (resp., $k \epsilon_{r}$ ) be a copy of $k$, let $\boldsymbol{Z} e_{r}$ (resp., $\boldsymbol{Z} \epsilon_{r}$ ) be a copy of $\boldsymbol{Z}$, let $A_{1}:=\oplus_{r \in A} \boldsymbol{Z} e_{r}$, let $A_{2}:=\oplus_{r \in A}$ $\boldsymbol{Z} \epsilon_{r}$, and let $B:=\boldsymbol{Z} e$, where $e$ is another symbol. Let $v_{0}: A_{1} \oplus A_{2} \oplus B \longrightarrow A_{1} \oplus B$ be the projection, let $w_{0}: A_{1} \oplus A_{2} \oplus B \longrightarrow A_{2} \oplus B$ be the projection, and let $u_{0}: A_{1} \oplus A_{2} \oplus B \longrightarrow B$ be the projection. Let $k$ be a field, let $k\left[X ; A_{1} \oplus A_{2} \oplus B\right]$ be the group ring of $A_{1} \oplus A_{2} \oplus B$ over $k$, and let $K$ be the quotient field of $k\left[X ; A_{1} \oplus A_{2} \oplus B\right]$. For $f=\sum a_{i} X^{\alpha_{i}} \in k\left[X ; A_{1} \oplus A_{2} \oplus B\right]$ with $a_{i} \neq 0$ for every $i$, and $\alpha_{i} \neq \alpha_{j}$ for every 
$i \neq j$, we set $v(f):=\min _{i} v_{0}\left(\alpha_{i}\right)$, then $v$ defines a valuation $v$ on $K$ with value group $A_{1} \oplus B$. Similarly, we have valuations $w$ and $u$ on $K ; v: K \longrightarrow\left(A_{1} \oplus B\right) \cup\{\infty\}$, $w: K \longrightarrow\left(A_{2} \oplus B\right) \cup\{\infty\}, u: K \longrightarrow B \cup\{\infty\}$. Let $V$ (resp., $W, U$ ) be the valuation domain of $v$ (resp., $w, u$ ), and let $M^{\prime}$ (resp., $N^{\prime}, P_{0}^{\prime}$ ) be the maximal ideal of $V$ (resp., $W, U)$, set $D:=V \cap W$, and set $M:=M^{\prime} \cap D, N:=N^{\prime} \cap D$, and $P_{0}:=D \cap P_{0}^{\prime}$. Then $M^{\prime}=M V, N^{\prime}=N W$ and $P_{0}^{\prime}=P_{0} U$. Moreover, $D$ is a Prüfer domain with exactly two maximal ideals $M$ and $N$. Clearly, $P_{0}$ is a height one prime ideal of $D$ with $P_{0} \subseteq M \cap N$.

Lemma 1 ([M1, Lemmas 2 and 4]). (1) $D$ is a Bezout domain.

(2) $\overline{\mathrm{F}}(D) \backslash \mathrm{F}(D)=\{K\}$.

(3) Every star operation $\star$ on $D$ can be uniquely extended to a semistar operation on $D$.

Lemma 2 ([M4, Example 1]). Every non-zero convex subgroup of $A_{1}$ is generated by a subset of $\left\{e_{r} \mid r \in A\right\}$. And, for every $r \in A$, the subgroups $L_{r}=\left\{k_{1} e_{r_{1}}+\cdots+\right.$ $k_{n} e_{r_{n}} \mid r_{1}<\cdots<r_{n} \leq r$, every $\left.k_{i} \in \boldsymbol{Z}\right\}$ and $L_{r}^{\prime}=\left\{k_{1} e_{r_{1}}+\cdots+k_{n} e_{r_{n}} \mid r_{1}<\cdots<\right.$ $r_{n}<r$, every $\left.k_{i} \in \boldsymbol{Z}\right\}$ are convex subgroups of $A_{1}$. The set $\left\{L_{r}, L_{r}^{\prime} \mid r \in A\right\} \cup\left\{\{0\}, A_{1}\right\}$ is the set of all convex subgroups of $A_{1}$.

Lemma 3. (1) For every $r \in A$, set $H_{r}=\left\{k_{1} e_{r_{1}}+\cdots+k_{n} e_{r_{n}} \mid r_{1}<\cdots<r_{n} \leq r\right.$, every $\left.k_{i} \in \boldsymbol{Z}\right\}$, and set $H_{r}^{\prime}=\left\{k_{1} e_{r_{1}}+\cdots+k_{n} e_{r_{n}} \mid r_{1}<\cdots<r_{n}<r\right.$, every $\left.k_{i} \in \boldsymbol{Z}\right\}$. Then the set $\left\{H_{r}, H_{r}^{\prime} \mid r \in A\right\} \cup\left\{A_{1}\right\} \cup\left\{\{0\}, A_{1} \oplus B\right\}$ is the set of all convex subgroups of $A_{1} \oplus B$

(2) If $Q$ is a non-zero prime ideal of $D$ with $Q \subseteq M \cap N$, then $Q=P_{0}$.

Proof. (1) The proof follows from Lemma 2.

(2) Suppose that $Q \supsetneqq P_{0}$. Take an element $d \in Q \backslash P_{0}$. Set $v(d)=\sum a_{i} e_{i}+c e$ with the $a_{i}, c \in Z$. Since $d \notin P_{0}$, we have $c=0$. Set $x:=X^{\sum a_{i} e_{i}}$. Then $v(d)=v(x)=\sum a_{i} e_{i}$. Since $w(x)=0$, we have $x \in Q \backslash N$, hence $Q \nsubseteq N$; a contradiction.

Lemma 4. $D$ has an infinite number of semistar operations $\star$ with $D^{\star}=D$.

Proof. By Lemma $3(1)$, we have $\operatorname{dim}(D)=\infty$. By Lemma $3(2), P_{0}$ is the largest prime ideal of $D$ contained in $M \cap N$. Since $\left|\operatorname{Spec}\left(\frac{D}{P_{0}}\right)\right|=\infty$. D has an infinite number of star operations by Theorem 1, that is, $|\operatorname{Star}(D)|=\infty$. Every member $\star \epsilon$ $\operatorname{Star}(D)$ can be uniquely extended to a semistar operation $\star^{\prime}$ on $D$ (Lemma $1(3)$ ). Since $D^{\star^{\prime}}=D$, the proof is complete.

Lemma 5. (1) $\star_{\{M, N\}}=\mathrm{d}$.

(2) Let $P, Q$ be incomparable prime ideals of $D$. Set $\star=\star_{\{P, Q\}}$, and set $T=D_{P} \cap D_{Q}$. Then $E^{\star}=E T$ for every $E \in \mathrm{F}(D)$.

Proof. (1) Let $E \in \mathrm{F}(D)$ and let $x \in E^{\star}=E D_{M} \cap E D_{N}$. We have $x=\frac{e_{1}}{s}=\frac{e_{2}}{t}$, where $e_{1}, e_{2} \in E, s \in D \backslash M$ and $t \in D \backslash N$. Then $(s, t)=D$, hence $1=s d_{1}+t d_{2}$ with $d_{1}, d_{2} \in D$. Then $x=s x d_{1}+t x d_{2}=e_{1} d_{1}+e_{2} d_{2} \in E$, hence $E^{\star}=E$, and hence $\star=$ 
d.

(2) Set $\Delta:=\{P, Q\}$. We may assume that $P \subseteq M$ and $Q \subseteq N$. Set $P D_{P} \cap T=$ $M_{1}, Q D_{Q} \cap T=N_{1}$. $T$ is a Prüfer domain with exactly two maximal ideals $M_{1}, N_{1}$. Further $T_{M_{1}}=D_{P}, T_{N_{1}}=D_{Q}$. Obviously $D^{\star}=T$. Let $J$ be a non-zero ideal of $T$. As in (1), $J=J T_{M_{1}} \cap J T_{N_{1}}=J D_{P} \cap J D_{Q}$. Let $I$ be a non-zero ideal of $D$. Then $I^{\star}=(I D)^{\star}=(I T)^{\star}=I T$, i.e., $I^{\star}=I T$.

Lemma 6. Let $\Delta=\left\{P_{\lambda} \mid \lambda \in \Lambda\right\}$ with every $P_{\lambda} \subseteq M$, let $\star=\star_{\Delta}$, and set $P:=\cup_{\lambda} P_{\lambda}$.

(1) We have $D^{\star}=\cap_{\lambda} D_{P_{\lambda}}=D_{P}$.

(2) If $P=M$, then, for every prime ideal $Q$ of $D$ with $Q \varsubsetneqq M$, we have $Q \subseteq P_{\lambda}$ for some $\lambda$.

Proof. (1) Let $x \notin D_{P}$, and set $m:=\frac{1}{x}$. Since $D_{P}$ is a valuation domain, we have $m \in P D_{P}$, and $s m \in P$ for some $s \in D \backslash P$. Then $s m \in P_{\lambda}$ for some $\lambda$. Since $1=m x=s m \frac{x}{s} \in P_{\lambda} \frac{x}{s}$, we have $\frac{x}{s} \notin D_{P_{\lambda}}$, hence $x \notin D_{P_{\lambda}}$. Therefore $\cap_{\lambda} D_{P_{\lambda}}=D_{P}$.

(2) Take an element $d \in M \backslash Q$. Then we have $\frac{d}{1} \in P_{\lambda} D_{M}$ for some $\lambda$ and $\frac{d}{1} \notin Q D_{M}$. It follows that $P_{\lambda} D_{M} \supseteq Q D_{M}$. Hence $P_{\lambda} \supseteq Q$.

Lemma 7. Let $\Delta_{1}=\left\{P_{\lambda} \mid \lambda \in \Lambda\right\}$ with every $P_{\lambda} \subseteq M$, let $\Delta_{2}=\left\{Q_{\sigma} \mid \sigma \in \Sigma\right\}$ with every $Q_{\sigma} \subseteq N$, set $\Delta=\Delta_{1} \cup \Delta_{2}$, and let $\star=\star_{\Delta}$. Set $P=\cup_{\lambda} P_{\lambda}$, and set $Q=\cup Q_{\sigma}$.

(1) $D^{\star}=\cap_{\lambda} D_{P_{\lambda}} \cap_{\sigma} D_{Q_{\sigma}}=D_{P} \cap D_{Q}$.

(2) $D^{\star}=D$ if and only if $P=M$ and $Q=N$.

Proof. (1) follows from Lemma 6 (1).

(2) $T:=D_{P} \cap D_{Q}$ is a Prüfer domain with maximal ideals $P D_{P} \cap T$ and $Q D_{Q} \cap T$. Assume that $D^{\star}=D$. Then $T=D$ by (1). Hence $P D_{P} \cap D=M$ and $Q D_{Q} \cap D=N$. Hence $P=M$ and $Q=N$.

Lemma 8. Let $X_{0}$ be the set of spectral semistar operations $\star$ with $D^{\star}=D$. Then $\left|X_{0}\right|=4$.

Proof. We confer Lemmas 5, 6 and 7. Let $\star \in X_{0}$, and let $\star=\star_{\Delta}$. By Lemma 6 (1), we have $\Delta=\Delta_{1} \cup \Delta_{2}, \Delta_{1}=\left\{P_{\lambda} \mid \lambda \in \Lambda\right\}$ with every $P_{\lambda} \subseteq M$ and $\Delta_{2}=\left\{Q_{\sigma} \mid \sigma \in \Sigma\right\}$ with every $Q_{\sigma} \subseteq N$. Set $P:=\cup_{\lambda} P_{\lambda}$ and $Q:=\cup_{\sigma} Q_{\sigma}$. By Lemma 7, we have $P=M$ and $Q=N$. We have the following four cases: (1) $\Delta_{1} \ni M$ and $\Delta_{2} \ni N$, (2) $\Delta_{1} \not \ngtr M$ and $\Delta_{2} \ni N$, (3) $\Delta_{1} \ni M$ and $\Delta_{2} \not \ni N$, (4) $\Delta_{1} \not \ni M$ and $\Delta_{2} \not \supset N$.

The case (1): $\star=\mathrm{d}$.

The case (2): We may assume that $\Delta_{1}$ is the set of all prime ideals $P^{\prime}$ of $D$ with $P^{\prime} \varsubsetneqq M$. Then $M^{\star}=D$ and $N^{\star}=N$.

The case (3): We may assume that $\Delta_{2}$ is the set of all prime ideals $Q^{\prime}$ of $D$ with $Q^{\prime} \varsubsetneqq N$. Then $M^{\star}=M$ and $N^{\star}=D$.

The case (4): We may assume that $\Delta_{1}$ (resp., $\Delta_{2}$ ) is the set of all prime ideals 
of $D$ with $P^{\prime} \varsubsetneqq M$ (resp., $\left.Q^{\prime} \varsubsetneqq N\right)$. Then $M^{\star}=D$ and $N^{\star}=D$.

Lemmas 4 and 8 imply the following,

There is an infinite number of non-spectral semistar operations $\star$ on $D$ with $D^{\star}=D$.

\section{References}

[H] E. Houston, Integrally closed domains which admit only finitely many star operations, The Booklet of Abstracts of The Conference "Commutative Rings and their Modules, 2012", Italy.

[M1] R. Matsuda, Note on the number of semistar operations, VIII, Math. J. Ibaraki Univ. 37 (2005), 53-79.

[M2] R. Matsuda, Commutative Semigroup Rings, 2nd. ed., Kaisei Publishing, Tokyo, 2006.

[M3] R. Matsuda, Note on spectral semistar operations, Bull. Allahabad Math. Soc. 25 (2010), 149-156.

[M4] R. Matsuda, Note on spectral semistar operations of finite type, JP J. Algebra, Number Theory and Applications 22 (2011), 225-231.

[P] G. Picozza, Star operations on overrings and semistar operations, Comm. Alg. 33 (2005), 2051-2073. 